Slavica

bruxellensia

\section{Slavica bruxellensia}

Revue polyphonique de littérature, culture et histoire

slaves

9 | 2013

Érotisme

\title{
Interdit et transgression dans Valérie ou la semaine des merveilles de Vítězslav Nezval
}

\author{
Sophie Teulière
}

\section{OpenEdition}

\section{Journals}

Édition électronique

URL : http://journals.openedition.org/slavica/1341

DOI : 10.4000/slavica.1341

ISSN : 2034-6395

\section{Éditeur}

Université libre de Bruxelles - ULB

\section{Référence électronique}

Sophie Teulière, «Interdit et transgression dans Valérie ou la semaine des merveilles de Vítězslav Nezval », Slavica bruxellensia [En ligne], 9 | 2013, mis en ligne le 15 avril 2013, consulté le 25 mai 2020. URL : http://journals.openedition.org/slavica/1341; DOI : https://doi.org/10.4000/slavica.1341

Ce document a été généré automatiquement le 25 mai 2020.

\section{(c) $($ ) $\odot \odot$}

Les contenus de Slavica bruxellensia sont mis à disposition selon les termes de la Licence Creative Commons Attribution - Pas d'Utilisation Commerciale - Pas de Modification 3.0 France. 


\title{
Interdit et transgression dans Valérie ou la semaine des merveilles de Vítězslav Nezval
}

\author{
Sophie Teulière
}

\section{Introduction}

1 Il suffit d'un rapide coup d'oeil en arrière pour se rendre compte et ce, de façon indubitable, que l'aspect de notre société moderne ayant le plus été influencé par le surréalisme est celui des mœurs amoureuses et de la vie sexuelle. Gérard Legrand l'affirme dans sa contribution à l'ouvrage collectif La femme surréaliste: «La longue transformation des pratiques et donc des représentations mentales relevant de l'érotique pendant le demi-siècle écoulé est inconcevable, mieux : inimaginable sans le surréalisme. $»^{1}$

2 C'est d'ailleurs bien ce que met en exergue l'exposition internationale du surréalisme se tenant, en 1959, dans les locaux de la galerie Cordier à Paris et dont André Breton rédige, avec d'autres, le catalogue. Ainsi, dans son avis intitulé "Aux visiteurs », il définit l'érotisme comme un principe unificateur du mouvement surréaliste :

Le plus grand commun diviseur des artistes surréalistes et de leurs œuvres c'est l'érotisme. Par delà leur extrême disparité de moyens et d'aspect, ce qui, dans leur ensemble, caractérise et qualifie les œuvres surréalistes, ce sont, au premier chef, leurs implications érotiques. ${ }^{2}$

Cependant, avant 1957, c'est-à-dire avant l'ouvrage de Georges Bataille intitulé $L^{\prime}$ 'Érotisme ${ }^{3}$, on ne trouve pas de texte théorisant de façon claire la notion d'érotisme ; c'est en un mot directement emprunté à la psychanalyse que les surréalistes placent leurs espoirs : la sexualité.

De janvier 1928 à août 1932, de nombreux membres du groupe surréaliste parisien se livrent avec la plus grande franchise - du moins, c'est ce qui est exigé de chacun d'eux à une série d'entretiens ayant pour thème la sexualité. Les séances abordent des 
problèmes aussi divers que l'onanisme, l'homosexualité masculine ou les fantasmes pervers. À $1000 \mathrm{~km}$ de là, à Prague, les figures de proue de l'avant-garde tchécoslovaque ne sont pas en reste. En 1933, un an avant la fondation par Vítězslav Nezval du groupe surréaliste pragois (dont Karel Teige sera le principal théoricien), Bohuslav Brouk, futur collaborateur du groupe, publie plusieurs œuvres abordant la sexualité humaine, comme sa très intéressante Psychoanalytická Sexuologie (Sexologie psychanalytique $)^{4}$, prouvant que les préoccupations des surréalistes parisiens sont également au cœur des réflexions de l'avant-garde tchèque. Déjà, en 1931, des collages réalisés par le plasticien Jindřich Štyrský, mettant en scène aussi bien des sexes féminins et masculins que des planches anatomiques des dits organes génitaux viennent illustrer un court récit de Nezval : Sexuální nocturno: přiběh demaskované iluse (Nocturne Sexuel : histoire d'une illusion démasquée) $)^{5}$. Publié dans la très particulière collection « $69 »^{6}$ dirigée par Štyrský, ce texte narre les premiers émois adolescents de l'auteur ; ses masturbations à la limite de la transe, habitées par des têtes de femmes désolidarisées de leur corps, ainsi que la perte de sa virginité dans les bras d'une prostituée de la petite ville de T. Dans le texte de Nezval, comme dans les œuvres de Štyrský et de leur comparse Marie Čermínová dite Toyen, le fait sexuel est au cœur d'une rhétorique qui caractérise la production littéraire et artistique poétiste puis surréaliste des années 1930. Chez ces artistes, la sexualité en tant qu'expérience intérieure est à la base d'une remise en question de l'homme et c'est précisément cela que Bataille nomme "érotisme »: "L'érotisme est dans la conscience de l'homme ce qui met en lui l'être en question. L'expérience intérieure de l'érotisme demande de celui qui la fait une sensibilité non moins grande à l'angoisse fondant l'interdit, qu'au désir menant à l'enfreindre. $»^{7}$

5 Ce que proposent les surréalistes, c'est d'échapper à la méconnaissance de soi en se tournant vers les mouvements érotiques qui terrifient l'individu (car là où il $\mathrm{y}$ a souci de reproduction et donc de vie, il y a aussi mort à plus ou moins longue échéance) et c'est ainsi que Bataille résume cette dualité essentielle : «L'érotisme est l'approbation de la vie jusque dans la mort. $»^{8}$

6 L'un des textes de Nezval, Valerie a týden divů(Valérie ou la semaine des merveilles, 1932), paraît emblématique de cette quête de soi bien qu'à première vue il n'apparaisse que comme un pastiche plus ou moins divertissant d'un genre cher aux surréalistes: le roman gothique. Une lecture attentive permet de mettre en évidence une réflexion extrêmement riche et originale sur les principes qui fondent l'être, prisonnier d'éternelles tensions aussi fascinantes qu'angoissantes.

\section{Le gothique, feinte futilité et véritables enjeux :}

7 Les années 1930 apportent un véritable changement au niveau des conceptions artistiques des poétistes pragois : l'attitude, les inspirations, les thèmes abordés par les artistes d'avant-garde se recentrent peu à peu sur l'individu dans toute sa complexité psychique. En effet, l'un des aspects clés faisant la transition entre poétisme et surréalisme en Tchécoslovaquie réside justement dans le remplacement de l'idéologie poétiste par le sombre monde de l'inconscient et de l'inconnu. C'est seulement à partir du moment où le poétisme accepte l'idée que la nature du drame inspirant l'horreur peut être porteuse de beauté que les avant-gardes tchèques entrent de plain-pied dans la phase de développement du surréalisme. Ainsi, à la première du Nosferatu de Murnau 
à Prague, Nezval s'exclame : «En art, l'horreur est charmante (...), en art, l'horreur doit être plus que de l'horreur, elle doit être poésie si on ne la confond pas avec la lecture des crimes dans les tabloïds. $»^{9}$

Avec le manifeste du surréalisme en 1924, les surréalistes ont commencé à réévaluer le genre gothique qu'ils tenaient comme réelle «antithèse " de la prose psychologique réaliste. En 1931, Antonin Artaud publie sa traduction et adaptation du Moine de Matthew Gregory Lewis, l'un des romans gothiques les plus populaires de l'histoire. Nezval aimait tant le roman anglais qu'il en avait commandé une traduction personnelle en tchèque et la position qu'il exprime dans son avant-propos à Valérie $\mathrm{y}$ fait écho: «J'ai écrit ce livre parce que j'ai l'amour du mystère qui est à la base des vieux contes, des superstitions et de tels livres romantiques, composés en caractères gothiques, qui m'ont fait parfois des clins d'œil sans consentir à me livrer leur contenu. $»^{10}$

9 Il est bien évidemment impossible de passer à côté des similitudes que présente Valérie par rapport à l'œuvre de Lewis. Nezval, dans ce récit « qui frôle le ridicule et la futilité » [Valérie, 7] ${ }^{11}$, emprunte de nombreuses situations, thèmes et personnages au roman anglais : on y trouve l'usage de potions simulant la mort, de déguisements, des relations incestueuses, des fratries séparées à la naissance. Même l'histoire de l'infortunée mère de Valérie, tombée enceinte alors qu'elle est emprisonnée dans un couvent, rappelle le sort d'Agnès qui, pour sauver son honneur prend le voile mais s'aperçoit quelques jours après être rentrée dans les ordres qu'elle attend un enfant. La tentative de Gratian pour déflorer Valérie est une réécriture du viol d'Antonia par Ambrosio dans les souterrains du monastère, et même si Nezval a décomposé le personnage d'Ambrosio en deux : le putois et Gratian, le trio Elsa-Gratian-Valérie correspond tout à fait au triangle amoureux Ambrosio-Matilda-Antonia.

D'autre part, Nezval reprend un certain nombre d'archétypes du roman gothique : le putois est le scélérat repoussant dont les traits difformes n'empêchent pourtant pas son pouvoir de séduction d'agir: "Je vois qu'en moins de deux il a pris beaucoup de pouvoir sur vous! » [Valérie, 100] constate Orlík. Dès lors qu'il a jeté son dévolu sur une victime, celle-ci n'a plus aucune chance, elle devra recourir à une aide extérieure afin de se soustraire à l'emprise du monstre. Mais le putois est aussi la représentation d'un personnage privilégié de l'esthétique gothique, dans la mesure où il représente l'infernale quête d'immortalité : le vampire. Celui-ci aspire le flux vital des autres êtres vivants pour prolonger sa vie ou sa jeunesse. "Vous n'avez point changé depuis quarante ans " [Valérie, 52], s'exclame la grand-mère de Valérie en apercevant pour la première fois le connétable, tendant ainsi à confirmer le caractère hors du commun de celui-ci. Bien qu'il se défende de tout pouvoir surnaturel : « Il ne faut pas m'attribuer je ne sais quel pouvoir magique» [Valérie, 55], une aura d'étrangeté entoure les mystérieux rituels auxquels il s'adonne afin de repousser un peu plus l'échéancier de la vie :

Valérie put distinguer, au-dessus de l'épaule nue d'Edwige, les contours de la silhouette noire et deviner le mouvement spasmodique de sa gorge, qui ingurgitait les flots d'amour et de plaisir répandus dans les veines de la jeune femme par le baiser interminable de son époux, où son esprit se diluait comme dans un rêve.

[Valérie, 92]

11 Mais l'un des aspects les plus spécifiques du vampire est son rapport au désir sexuel. Désir qui, dans les textes gothiques, prend comme support le corps conçu par la symbolique religieuse judéo-chrétienne, où ce dernier étant d'une part fait à l'image de 
Dieu, sera intact lors de la résurrection et d'autre part étant matériel, est sujet à corruption par le temps. Il en résulte un corps double, séparé entre âme immatérielle et enveloppe charnelle. Dans Valérie, cette conception largement répandue dans les textes gothiques où le désir est forcément lié à l'animalité, sinon à la présence du mal, est poussée à son paroxysme en posant un masque de putois sur le visage du connétable lubrique. Ses appétits sexuels sont ainsi caractérisés par leur monstruosité, et de ce fait, la métamorphose est complète; la bestialité à laquelle se rattache le désir est clairement portée en étendard par le roman.

L'espace dévolu à l'intrigue dans Valérie est extrêmement spécifique. En effet, la jeune fille n'est pas attachée à un couvent, à un château ou à une cathédrale, comme c'est majoritairement le cas dans les romans gothiques traditionnels, où les édifices témoins d'un passé religieux ou d'un pouvoir politique sont les théâtres des plus étranges phénomènes. La demeure noire du roman de Nezval est la maison de la grand-mère, enjeu de son marché avec le putois qui en revendique la propriété. C'est un lieu clos où règne un pouvoir absolu, capable d'une violence sans frein, mais en apparence légitime : une honorable vieille femme censée protéger la jeune fille, un missionnaire, garant de sa virginité et du respect des mœurs religieuses. Ces personnages qui devraient être rassurants, puisqu'ils incarnent l'ordre social et familial, s'avèrent au contraire le plus grand obstacle qui s'impose à la jeune héroïne, à l'image de la maison qui dresse au soleil sa force légitime mais cache sa face nocturne. Cette force maléfique s'incarne dans les souterrains, c'est dans ces espaces labyrinthiques où la fuite semble impossible que le destin des personnages se scelle, rappelons que le viol d'Antonia par Ambrosio a lieu dans les souterrains du monastère, et que c'est là même que Valérie est quasiment violée par celui qu'elle croit être son père.

La présence d'une structure souterraine dans la plupart des romans gothiques est une ressource dramatique presque illimitée. Par ailleurs, ces lieux possèdent une véritable richesse symbolique: la structure implique deux sortes de trajectoire: celle qui descend vers l'enfer et celle qui monte vers le salut. Cependant il existe un lieu encore plus noir dans ce labyrinthe inquiétant : la crypte, lieu de confusion entre vie et mort. C'est là, dans ce lieu non béni, que les vivants sont déposés dans des caveaux comme des morts et que les morts se lèvent comme des vivants. Échappant à la juridiction divine, la crypte est un espace privilégié des forces infernales. Le doute y subsiste toujours, il devient impossible de séparer les deux mondes: «J'en suis encore à me demander si vous êtes réellement vivante.» [Valérie, 148] Ce que renferment les souterrains, c'est ce qui est défendu par les hauts murs de la demeure noire : l'entrée du monde infernal ou un secret inavouable et terrible qu'il faut à tout prix défendre. Ce que Valérie découvre dans les souterrains, c'est la vérité sur les adultes qui l'entourent. Pour la jeune fille, la descente infernale correspond à une prise de conscience de la duplicité du monde. Il se dégage ici un aspect extrêmement important de l'esthétique de Nezval: le décor gothique n'est pas une fin en soi, il est doublé d'un second sémantisme, psychique cette fois. L'espace dans lequel Valérie est enfermée n'est pas la maison, mais bel et bien son propre subconscient métaphorisé ici par la thématique du rêve. Mais le genre gothique n'intéresse pas seulement Nezval pour sa capacité à reproduire les sinuosités de l'âme humaine. Il est également un moyen d'extraire les souvenirs comme un aimant: «Le fantastique dans Nosferatu repose sur la réalité, sur de vrais objets, des objets obsolètes ainsi capables de toucher notre mémoire et nos rêves, et dans la réalité demeure le charme surréaliste de ce film. $»^{12}$ 


\section{La nuit, espace d'union et de déchirement :} psychique complètement irrationnel, empruntant librement au genre de la littérature populaire tout ce qui appartient aux régions les plus reculées de l'inconscient humain ${ }^{13}$. Fortement influencé par la technique plastique des collages surréalistes, et notamment par ceux de Max Ernst qu'il a vu exposés à Prague dans les années 1930 et à Paris, le titre même de son roman suggère un collage entre le travail d'Ernst dans Une semaine de bonté et Alice au pays des merveilles de Lewis Carroll. D'après Guiseppe Dierna (auteur de la traduction anglaise du roman de Nezval), Valérie prend sa source dans le romancollage d'Ernst intitulé Rêve d'une petite fille qui voulut entrer au Carmel, dont la narration s'effectue en une succession de 79 collages sous-titrés de façon brève et contant la quête du céleste fiancé par la jeune Marceline-Marie. Dès les premières pages, le rêve de la petite fille nous rappelle l'atmosphère incestueuse englobant l'histoire de Valérie, ainsi que la lubricité ecclésiastique sans cesse présente :

[Le Père:] Votre baiser me semble adulte, mon enfant. Venu de Dieu, il ira loin.

Allez, ma fille, allez en avant et... comptez sur moi !

[Marceline-Marie :] Ma tenue me semble indécente, papa, en présence du Père

Dulac. L'épreuve la plus délicate pour une Enfant de Marie...

[Le R. P :] La joie sera près de vous, mon enfant !

[Le Père :] Laissez-moi pleurer et... serrer cette main qui serait condamnée à végéter dans une cellule! Écoutez-moi, mon enfant: la calvitie vous guette.

Marceline-Marie se fend en deux (Angoisse et cris). ${ }^{14}$

thématique du double - que l'on retrouve dans la structure des romans gothiques - permet à Ernst d'introduire une réflexion sur la recherche de l'identité : «Qui suisje? Moi, ma sœur ou ce scarabée obscur ? $\aleph^{15}$ En baptisant son héroïne d'un prénom composé, il crée un trouble que la jeune fille ne peut résoudre à l'intérieur de son rêve qu'en se représentant elle-même sous les traits de personnes distinctes mais extrêmement semblables, deux sœurs, l'une Marceline, l'autre Marie : «moi » et ma «sœur ». Ce qui nous permet d'envisager le fait que, dans le roman de Nezval, Valérie et Orlík ne sont également que deux moitiés d'un tout, car l'histoire de Valérie atteint son point culminant lors de la réunification de cet être originel. Cette considération inscrit le roman dans la philosophie platonicienne de la recherche de la partie manquante, afin de recréer l'hermaphrodite primordial composé d'un homme et d'une femme. Ainsi, on peut affirmer que Valérie voit en Orlík plus que son jumeau, elle trouve en lui son double, la partie qui lui manquait jusque-là pour sortir du rêve. Comme une clé, Orlík lui permet de déverrouiller la porte de sortie. Plusieurs fois, au cours du roman, le lien qui unit les deux jeunes gens, tout ambigu qu'il soit, frôle l'inceste puisqu'il s'exprime en premier lieu par les vecteurs propres à la relation amoureuse. Valérie est désemparée devant les sentiments nouveaux qu'elle éprouve à l'égard de cet inconnu qui a décidé de la protéger, mais petit à petit, ceux-ci s'estompent : «Elle sentait diminuer l'ascendant que le jeune homme avait si fortement exercé sur elle. » [Valérie, 59]

Puisqu'au vu des révélations de la grand-mère sur la généalogie de sa famille, la réunion ne peut pas s'effectuer sur un plan amoureux et physique entre les deux personnages, c'est sur un plan spirituel et métaphysique qu'elle agit. Tout d'abord en permettant à Valérie de s'extraire, dans les derniers chapitres, du monde onirique dans

Slavica bruxellensia, 9 | 2013 
lequel elle était plongée, puis à la fin du roman, sur le terrain de la réalité, lorsque les songes se sont évanouis, c'est d'une seule voix qu'elle et Orlík entonnent un chant s'adressant aux amoureux, hommes et femmes.

C'est en tant qu'androgyne réunifié qu'ils s'expriment de concert, et c'est à ce moment que prend fin la quête identitaire de Valérie, dans la sublimation du conflit et dans l'innocence du paradis perdu de l'enfance.

18 Mais cette recherche de la moitié perdue n'est pas, dans l'œuvre de Nezval, limitée à Valérie. En effet, elle est aussi évoquée, à maintes reprises, dans les poèmes du recueil La Femme au pluriel, notamment dans le poème "Météore" où les femmes sont comparées à ces corps célestes qui s'embrasent « comme un chapelet de lumières aux filaments brûlés ${ }^{16}$. Le poème se conclut sur une énumération, comme c'est souvent le cas pour les poèmes de cette période, de comparaisons entre la rencontre du poète et de la femme aimée, et celle d'objets concrets si différents les uns des autres que leur seul lien est en réalité oxymorique :

Mes nuits comme les rendez-vous de mouches et de lampions

Au-dessus d'un tas de plumes

Comme les rendez-vous des tours et des femmes nues

Comme les rendez-vous de trompettes avec des nids d'oiseau

Comme les rendez-vous des terrasses avec des tonnerres

grondants

Comme les rendez-vous des pelles avec de l'eau de source

Comme les rendez-vous d'une balance de pharmacien avec

l'éclat du rubis ${ }^{17}$

Ces objets, pourtant de nature différente, s'unissent dans le giron de la nuit, comme l'homme s'unit à la femme qui lui est destinée. La nuit est un thème récurent dans l'œuvre de Nezval: la quasi-intégralité des aventures de Valérie se déroulent pendant la nuit. Usant souvent de l'oxymore pour traduire tous les visages de cette période qu'il affectionne, il tente d'exprimer le lien intime qu'il entretient avec elle. L'obscurité décore ses rêves, inspire ses représentations mentales et sa poésie. Mais la nuit chez Nezval n'est pas complètement opaque. Au contraire, elle est perforée de points lumineux, et si ce n'est pas par la belle face argentée de la lune, ce sera par les étoiles, les lampes à pétrole dont la flamme vacille à chaque pas et qui créent cette atmosphère si chère à l'auteur, et enfin les lampadaires courbés le long des ponts dont la lumière se réfléchit dans l'eau noire de la Vltava. C'est d'ailleurs munie d'une lampe à pétrole que Valérie fait sa première apparition dans le roman. L'obscurité qui englobe alors la cour de la maison opère une modification de l'espace quotidien et, étonnée, la jeune fille remarque que « toute la cour était transformée ». [Valérie, 10] La nuit est donc en ellemême plus qu'une temporalité, un espace où les règles du jour ne s'appliquent plus et où il est impossible de savoir avec certitude si l'on se trouve sur le bord glissant qui sépare la conscience de la rêverie. C'est aussi un espace propice au déchaînement des passions amoureuses et dans le roman de Nezval, la sphère nocturne est fortement associée au plaisir sexuel, bien que du plaisir aucun personnage ne parvienne à en avoir réellement: Valérie échappe de justesse au viol, la nuit de noce d'Edwige se solde par une morsure qui l'ampute de plusieurs années de vie, les prostituées se refusent au putois... le seul acte sexuel poussé à son terme est le rapport ayant lieu entre la prétendue cousine de Valérie et le garçon de ferme, il est alors intéressant de noter que la scène a lieu en journée. Dans ce monde inversé, la signification symbolique de la nuit est renversée, l'acte sexuel ne peut y être poussé jusqu'à son terme : il reste un échec. 
20 La nuit chez Nezval présente donc une dualité essentielle, à l'image de son astre qui, d'un côté, semble protéger Valérie en la guidant à travers le dédale de ses aventures, mais de l'autre la condamne à errer dans une pénombre qu'elle peuple de projections terrifiantes. De ce fait, la lune est un élément extrêmement ambivalent : la face qu'elle montre est celle de Diane, déesse violente mais juste, protégeant les femmes qui forment son cortège, la face qu'elle cache est celle d'Hécate, belle et terrifiante, présidant aux ombres et aux morts. C'est elle qui suscite les cauchemars et les terreurs nocturnes, symboles des désirs secrets ou refoulés de l'inconscient. Elle personnifie de façon allégorique le processus automatique, en offrant un support particulièrement bienvenu aux vues modernes des surréalistes. Hécate est l'expression consubstantielle de la mort liée à la fascination pour la beauté féminine. À elle seule, elle résume la passion dévorante et la violence qu'elle signifie, elle est l'expression de l'érotisme dans la mort.

\section{La mort et le tabou du sang :}

21 Si la mort fascine l'auteur surréaliste, c'est parce qu'elle est profondément liée au scandale qui introduit dans l'ordre moral une rupture entre bien et mal et elle reste, en ce sens, l'occasion d'un dépassement. Le scandale dans les textes érotiques est nécessaire car il introduit une vue tragique qui s'exprime dans la formule de Bataille citée plus haut : «L'érotisme est l'approbation de la vie jusque dans la mort. ${ }^{18}$ Celle-ci ouvre un grand paradoxe primordial sur lequel, selon lui, repose toute la construction psychique humaine. Si l'érotisme se définit comme une expression particulière de la reproduction sexuée, il n'en est pas moins une « exubérance de la vie » ${ }^{19}$ qui n'est pas étrangère à la mort. Pour appuyer son raisonnement, il convoque la pensée du marquis de Sade qui se résume dans cette simple phrase : « Il n'est pas de meilleur moyen pour se familiariser avec la mort que de l'allier à une idée libertine. ${ }^{20} \mathrm{Ce}$ que Bataille aperçoit derrière cette affirmation (qui ne peut que choquer une morale qui voudrait voir en l'acte érotique une simple façon de célébrer la vie), c'est ce qu'il est possible de voir dans Valérie ainsi que dans l'ensemble des productions surréalistes : celles mettant en jeu l'érotisme se doublent obligatoirement d'images et de représentations qui l'associent directement à la mort ou, dans une moindre mesure à la souffrance engendrée par la peur de celle-ci.

22 L'érotisme est donc, d'une manière ambiguë, un fondement de l'être, ce qui crée une tension entre exaltation de la mort dans les jeux dramatiques et tragiques et exaltation de la vie dans la glorification du corps. L'être perdu dans cette tension qui le dissout met alors en évidence une dynamique entre deux catégories de pulsions, celles de vie et celles de mort.

23 La pulsion de vie est l'unité, son principe sous-jacent consiste en la conservation de l'être. Elle englobe la sexualité comme la recherche d'une cohésion et du maintien de celle-ci. Tendant à atteindre une certaine homéostasie selon le terme freudien, elle s'oppose à la décharge complète de l'excitation que représente la pulsion de mort. Cependant, Bataille introduit une nuance dans ce raisonnement : si l'être humain s'unit à l'autre, c'est qu'il est un être discontinu, séparé par un abîme vertigineux des autres êtres discontinus, et cherche dans l'acte sexuel à retrouver la continuité que seule la mort peut lui offrir. «Une continuité s'établit entre eux pour former un nouvel être à partir de la mort. ${ }^{21}$ Du point de vue de Bataille donc, la sexualité se tient du côté des 
liens du fait qu'elle cherche la continuité mais elle ne peut la trouver que dans la mort comme force primaire profondément pulsionnelle. Le surréalisme, effrayé autant que fasciné par ce côté obscur de l'érotisme, tente d'échapper à cette pulsion par la 《 compulsion à la synthèse $»^{22}$, un éternel retour vers l'unitaire, vers l'idéal platonicien de l'unité non-séparée. La plupart des textes surréalistes renvoient ainsi à cette jouissive tendance qu'éprouve l'homme à se retrouver au centre de la spirale où les choses ne vont pas vers un but central, mais restent dans la répétition, dans un statu quo qui tourne en rond. Dans cette illusion d'être sans être vraiment, où les événements sont sans avènement, où plus rien ne se passe, la pulsion de mort est une jouissance sadique mortifère qui se traduit par le désir non pas de chercher mais de plonger dans le néant de la spirale que définit le manque, le manque de l'autre, une partie perdue de soi.

Cette spirale de la pulsion correspond à celle du temps qui s'enroule sur lui même pour échapper à sa feinte linéarité, des ponts peuvent ainsi se créer entre les évènements, ponts dont la construction par l'esprit est motivée par certaines situations, certains mots et certains actes.

L'acte sexuel, dans lequel la pulsion trouve sa forme, représente la recherche du retour de l'absolu dans l'orgasme. À ce moment, le protagoniste vit la "petite mort », l'arrêt du temps, de la pensée, avant la reprise. Mais ce qui intéresse avant tout Nezval comme une grande partie des artistes surréalistes, c'est le rapport qu'entretient la femme - et non l'homme - à l'érotisme. Car la femme, en plus d'être l'objet du désir masculin, présente une sexualité mystérieuse et terrible placée sous le signe du sang: c'est seulement au terme du déchirement et de la rupture qu'elle accède au statut de femme qui lui permettra de joindre le mouvement elliptique des pulsions.

Le phénomène de la défloration est lié aux tabous concernant le cycle féminin comme naissance et renaissance. Un lien très fort se crée alors entre le sang des premières menstruations et celui versé lors du premier rapport. Dans les sociétés primitives, l'initiation des filles débute avec les premières menstruations, elles marquent un changement physiologique et commandent une rupture qui arrache la jeune fille à son monde familier, c'est le premier pas vers un nouvel état des choses.

Le sang concentre en lui les peurs ancestrales liées aux cycles de la vie et de la mort, de la naissance et du trépas. Le sang menstruel ainsi que celui de la virginité relèvent du cruor en latin, le sang qui coule par une émission pathologique et par un déversement de sécrétions en opposition au sanguis contenu dans un système clos et préservé de l'air qui le corrompt. Ainsi, le sang féminin est symboliquement associé au fluide quitté par la vie rappelant ainsi à l'humain le dépérissement et la pourriture de toute chose vivante.

Dans Valérie, la relation entre premières menstruations et début du parcours initiatique est très aisée à établir. En effet, le premier chapitre correspond à la découverte du saignement: "En regardant [sa cheville] de près, elle la vit, à sa grande frayeur, encerclée par un mince filet de sang. » [Valérie, 15] Tout comme les poules égorgées par le putois, Valérie perd son sang et devient donc une victime toute désignée.

Les aventures de Valérie sont donc, d'une certaine manière, provoquées par l'apparition des règles qui, violemment, la font sortir du monde des enfants pour la projeter dans celui bien plus inquiétant des adultes. Le rêve suggère la perte de repères qu'implique la découverte d'un monde nouveau en le métaphorisant. Valérie, qui 
jusqu'alors n'était pas assez "mûre » pour que sa grand-mère lui confie le secret des ses origines, est frappée de plein fouet par la duplicité des adultes et de leur univers :

Sois contente d'être encore une enfant.

Je ne lui suis plus, grand-mère. Justement, cette nuit... [Valérie, 17]

Le cinquième jour, celui marquant l'arrêt de l'écoulement sanguin, la chambre de Valérie est, pour la première fois inondée de soleil : «Le soleil inondait sa chambre et faisait jouer ses rayons sur les voilages.» [Valérie, 187] «Comme si rien ne s'était passé ", elle court rejoindre sa grand-mère au salon. Celle-ci est redevenue la vieille femme respectable qu'elle était au deuxième chapitre. Maintenant que le putois est définitivement mort, elle se livre à d'autres aveux permettant à Valérie de faire la lumière sur ses origines et de retrouver son frère Orlík. La notion de cyclicité est très présente tout au long du texte : cycle des événements avec la portée symbolique de la prophétie ( Jamais plus je ne te recevrai, à moins que par miracle mes cheveux ne partent tout seuls pour te ramener. " [Valérie, 207]), cycle de la femme qui se répète de génération en génération (" À dix-sept ans, exactement comme ta mère » [Valérie, 17]), succession des jours et des nuits, alternance entre rêve et cauchemar, etc. Nezval calque ainsi le temps du récit initiatique sur celui des premières menstruations, inscrivant le destin de son héroïne dans un parcours elliptique dont elle ne peut sortir.

Prisonnière, Valérie l'est aussi des hommes qui tentent de la posséder, et en ce sens, elle prend à plusieurs reprises les traits d'une victime de la concupiscence masculine. Victime, car c'est ici sa virginité qui est menacée et dont le mince filet rouge qui en découle est versé au sacrificateur comme une obole de sang. Et si la défloration est associée au sacrifice, c'est que, selon Bataille, l'homme (discontinu) est ramené à la continuité de l'être et à l'absence de particularités dans la mort qui lui offre l'illimité (mais en le privant du limité), l'infini qui appartient à la sphère sacrée : «Elle est voulue comme l'action de celui qui dénude sa victime, qu'il désire et qu'il veut pénétrer. L'amant ne désagrège pas moins la femme aimée que le sacrificateur sanglant l'homme ou l'animal immolé. $»^{23}$

31 Le sang sacrificiel a une valeur assez ambivalente, sujet à l'écoulement par une ouverture étrangère aux voies naturelles (béance où le souffle vital s'écoule pur et régénérant), il est partagé entre sanguis et cruor, entre sacré et profane. La notion de sacrifice n'est par ailleurs pas étrangère à cette première représentation par laquelle le meurtre prend la texture de la rédemption : celle d'une mort dont la finalité est un commerce avec le divin. La femme, dans les mains de celui qui l'assaille, est petit à petit dépossédée d'elle-même :

(...) Elle perd, avec sa pudeur, cette ferme barrière qui, la séparant d'autrui, la rendait impénétrable: brusquement elle s'ouvre à la violence du jeu sexuel déchaîné dans les organes de la reproduction, elle s'ouvre à la violence impersonnelle qui la déborde du dehors. ${ }^{24}$

Valérie repousse les assauts d'hommes plus âgés et leur échappe uniquement grâce au sortilège contenu dans ses boucles d'oreille. La différence d'âge introduit une idée de domination qui va de pair avec celle du sacrifice où le sacrificateur a autorité sur la victime : ici, sur le plan sexuel aussi bien que sur le plan social, la femme n'est plus une figure de l'altérité dans laquelle l'homme trouve sa moitié manquante, mais bel et bien un objet qu'il faut totalement annihiler pour le posséder. La sexualité féminine n'est plus une question de désir de la femme mais de supériorité physique ou sociale de l'homme et de son propre désir qu'il imprime de façon unilatérale. 
posséder la femme, Nezval évoque une sexualité que l'on peut qualifier de perverse, dans la mesure où elle traduit une satisfaction érotique bannie des mœurs sociales: Valérie est confrontée au viol à deux reprises par un prêtre, qui de ce fait rompt ses vœux, puis par celui qu'elle pense être son père; sa grand-mère elle aussi tente de la séduire. Le viol, et dans une plus forte mesure encore l'inceste, sont de puissants symboles de la perversité sexuelle portant atteinte au corps de la société. Cette sexualité qui se complait dans l'immoralité et la corruption des esprits et des corps, si elle était libérée, mettrait en danger la société :

Contre une société qui utilise la sexualité comme moyen pour réaliser une fin socialement utile, les perversions maintiennent la sexualité comme une fin en soi ; elles se placent ainsi en dehors du régime du principe de rendement et mettent en question sa base même. Elles établissent des relations libidineuses sur lesquelles la société doit jeter l'anathème, parce qu'elles menacent de renverser le processus de civilisation qui a transformé l'organisme en instrument de travail. ${ }^{25}$

La perversion et son rapport destructeur à la société nous rapproche de la vision du Marquis de Sade que les surréalistes ont érigée en grande figure libératrice ayant ébranlé le monde. Mais chez le Divin Marquis, ce sont les institutions qui sont considérées comme une perversion pour "l'espèce ", les surréalistes, eux, ont voulu la libération d'Éros comme force subversive : «Dans un monde d'aliénation, la libération d'Éros opérerait inévitablement comme force destructrice, fatale, comme la négation totale du principe qui gouverne la réalité répressive. $»^{26}$ Mais derrière cette apparente volonté de déstructurer le corps de la société, c'est encore plus le corps humain qui fait l'objet des attaques surréalistes. Les comportements sexuels relatés dans les textes surréalistes et qualifiés de pervers par la société qu'ils menacent, ne sont qu'un premier pas vers l'éclatement du corps humain lui-même. La sexualité, considérée d'un point de vue surréaliste, est indissociable de la violence, d'après Bataille, «à la base de l'érotisme, nous avons l'expérience d'un éclatement, d'une violence au moment de l'explosion $\aleph^{27}$. Cet éclatement entraîne la désintégration du corps s'opposant au mouvement de compulsion à la synthèse et rendant de ce fait impossible l'extraction de la spirale des pulsions.

\section{Perversion et destruction du corps :}

D'après Xavière Gautier, auteur d'un fascinant ouvrage sur le surréalisme, la force de ce mouvement a été d'avoir « inscrit dans ses prémices que l'art, comme la révolution, est une violence, un rapt et une métamorphose douloureuse du corps $»^{28}$. Écrire n'est bien évidemment pas un geste innocent, c'est un acte de violence car il y va du corps : « tant qu'on n'aura pas changé l'anatomie de l'homme actuel, il n'y aura rien de fait ni pour la poésie, ni pour aucune espèce réelle $»^{29}$, affirme Artaud dans une lettre adressée à Breton en 1968. Il faut rappeler que, onze ans plus tôt, dans sa radiodiffusion intitulée Pour en finir avec le jugement de Dieu, il avait déclaré la guerre aux organes: "Car liezmoi si vous voulez, mais il n'y a rien de plus inutile qu'un organe. $»^{30} \mathrm{En}$ disant cela, Artaud, plus que de lutter contre les organes, lutte contre l'organisme et par là tout le système théologique car c'est justement ce jugement de Dieu qui arrache le corps sans organe à son immanence (le plan de consistance propre au désir) et lui donne une signification et un sujet. ${ }^{31}$

Le corps est le corps,

il est seul 
et n'a pas besoin d'organes,

le corps n'est jamais un organisme,

les organismes sont les ennemis du corps,

(...)

Tout organe est un parasite. ${ }^{32}$

Pour les surréalistes, le mouvement révolutionnaire qu'ils revendiquent pleinement est celui de l'éclatement, du sacrilège et de la transgression; d'ailleurs, l'acte surréaliste par excellence est le plus gratuit et celui qui brise le plus d'interdits, puisqu'il consiste « revolver au poing, à descendre dans la rue et à tirer au hasard, tant qu'on peut dans la foule $»^{33}$.

Dalí (bien qu'il se soit séparé des surréalistes sur le plan politique), trouve un accent proprement surréaliste en observant une scène de la révolution espagnole dans laquelle il sent que se mêlent étroitement Éros et Thanatos : «L'Espagne martyrisée où s'élève une odeur d'encens, de chair de curé brûlée, de chair spirituelle écartelée mêlée à la senteur puissante de la sueur des foules forniquant entre elles et avec la Mort. ${ }^{34}$

Chair, fornication violence et jouissance, c'est bien le corps qui est en jeu dans la révolution surréaliste et qui, pourrait-on dire, en est l'enjeu. Idée parfaitement résumée par Artaud dans son post-scriptum au Théâtre de la cruauté : « Le théâtre, la danse, le chant, sont le théâtre des révoltes furieuses de la misère du corps humain. ${ }^{35}$

dit plus haut que par la violence des perversions sexuelles, les surréalistes portaient atteinte à la structure de la société en y introduisant un mal qu'elle rejette. Dans une visée tout à fait révolutionnaire, ils se posent en porte-à-faux avec la norme sans laquelle il est impossible de concevoir les dites perversions. Avant Freud, et encore aujourd'hui, le mot perversion désigne une déviation par rapport à la "normalité » d'un comportement; cependant, le père de la psychanalyse ne la reconnaît qu'en rapport avec la sexualité. ${ }^{36}$

Les surréalistes ne se contentent pas d'évoquer des comportements pervers pour ébranler les bases de la civilisation moderne. Afin de toucher à leur but plus profond qui est la déstructuration du corps lui-même, ils adoptent (inconsciemment ou pas) le comportement d'un individu pervers au sens psychanalytique du terme : une attitude d'appropriation de l'autre en tant qu'objet partiel dans le but de s'alimenter soi-même.

La déstructuration visée est celle de la représentation psychique du corps comme quelque chose qui n'est plus un Tout. Il est décomposé en morceaux qui devraient être liés ensemble (ma main, mon estomac, etc.), mais qui ne sont plus que des éléments libres que l'être ne peut pas maîtriser.

Un comportement psychique normal du développement humain est la recherche (vaine) de l'unité perdue (celle d'avec la mère), elle met en place la notion du Moi limité, du Moi en manque, manque de l'autre différent du moi. Le sujet - Moi différent de l'autre - se constitue en opposition à cet Autre extérieur. ${ }^{37}$ Si un rapport sexuel est fondamentalement un lien avec l'autre pour s'unir à l'endroit où cet autre existe comme entité différente, chez le pervers l'autre n'existe pas comme un sujet à part entière avec qui on se lie, mais comme un objet qu'on détruit car on s'en sert. La jouissance est le produit de cette possession de l'autre comme objet partiel. Dans la sexualité dite normale, il y demeure malgré tout un peu de cette dimension perverse : on s'unit avec l'autre, on le possède pour être entier.

En se soumettant ou en asservissant le corps de l'autre, le pervers le soumet, le partialise, le décompose en parties distinctes les unes des autres : le sexe, les bras, la 
bouche, les yeux, etc. C'est ainsi que Nezval procède dans son "Píseň písní » (Le Cantique des cantiques), sous le parcours du regard amoureux, le corps de la femme aimée est petit à petit décomposé, séparé en parties qui n'ont plus rien à voir avec le tout qu'elles formaient avant que le poète ne s'attache à son existence. Peu à peu, ces morceaux deviennent indépendants les uns des autres, comme si dans la séparation, ils retrouvaient une unité que leur accorde le regard de l'homme :

Ta bouche est une décoration vermeille

Devant laquelle on se découvre et se met au garde-à-vous

(...)

Tes mains sont des ciseaux avec lesquels tu découpes mon rêve ${ }^{38}$

La façon dont il métamorphose ces éléments désarticulés rappelle le travail pictural de Max Walter Svanberg qui crée des femmes hybrides d'animaux, de plantes ou d'objets en faisant se muer leur corps. La bouche est formée par un papillon, le buste par une poule, le sein par une tête d'oiseau dont le bec pointe vigoureusement en avant, les bras par des cous de cygnes... Leur ventre-miroir, leur corps de perles, leur buste en calice en font une mosaïque bizarre, les différents tessons du corps s'échangeant au gré de la fantaisie de l'artiste " obsédé par Son inévitable corps en fleurs, obsédé par Ses cheveux de danse de Saint-Guy qui tourbillonnent lourdement comme des cygnes ivres et noirs entre Ses nénuphars, obsédé par Ses joues bleu ciel, parées de papillons doubles, [il] sent un désir d'animal aveugle pour Son ventre éblouissant $\aleph^{39}$. De la même façon, Nezval métamorphose les éléments de la structure féminine qu'il a éclatée en animaux, en fleurs, en objets, en astres :

Ton sexe est un sifflet fendu de saule

Un œil aimant humide

Une libellule

Un mimosa

Ton sexe est une luciole au cœur d'un myriophylle ${ }^{40}$

Proches de la démarche du pervers, les surréalistes tentent de désarticuler le corps en signes, la perversion surréaliste sexualise: elle utilise les parties, dans l'usage du découpage, du collage, de l'anagramme. Elle fait du langage un substitut du corps. Alors, les surréalistes font avec les mots ce que le corps fait dans la sexualité, ce que, dans la sexualité, devient le corps découpé cherchant le fond unifiant: «Ce sont les mots qui font l'amour. $»^{41}$

Ce bouleversement, cette métamorphose du corps, se dessine dans chaque perversion et c'est sans doute son principal intérêt: faire que le corps ne soit plus corps naturel mais inventé dans le sens surréaliste du terme. Il ne s'agit pas d'une création ex nihilo car l'objet est préexistant, on en change seulement la destination, le sens donc la signification.

Dans les illustrations de Štyrsky pour Sexuální nocturno, tout est sexualisé, la bouche d'une femme, affublée de poils pubiens devient un sexe entrouvert, un traversin prolongé par les traits au crayon, tenu du bout des doigts par une jeune fille, n'est autre qu'un sexe d'homme en érection et plus loin, un sexe féminin monumental semble être l'entrée d'une caverne fabuleuse dans laquelle se génère une véritable armée de nourrices et de landaus.

La métamorphose et la multiplication des membres féminins peuvent alors être perçues comme des désirs de multiplier les possibilités érotiques et de reculer les limites de la jouissance. Elles deviennent l'expression de fantasmes où se manifeste la volonté de 
pulvériser les limites de la vie individuelle, le corps n'est plus le corps anatomique mais un foyer de désir.

Le scénario du pervers s'inscrit ici dans une certaine légitimité, l'artiste surréaliste tente de s'approprier l'autre pour exister. Exister dans le mouvement qui peut bien être la jouissance comme le tourment charnel ou psychique car ils sont tous deux opposés au vide qu'ils tentent désespérément de fuir.

Que chaque élément du corps soit sexualisé permet de ne pas les réduire à des instruments de labeur utilisés à des fins socialement utiles. Ainsi présentées, il semble que les perversions surréalistes atteignent les fins proposées par Herbert Marcuse, à savoir menacer de renverser le processus de civilisation qui a transformé l'organisme en instrument de travail..$^{42}$ La volupté serait ainsi, chez les surréalistes, opposée au travail : la main qui construit le monde contemporain est aussi celle qui fait jouir. Mais Bataille présente une opposition fondamentale entre travail et violence, "ce que le monde du travail, dit-il, exclut par des interdits est la violence $»^{43}$ et si l'on admet que le mouvement de l'amour porté à l'extrême est un mouvement de mort, alors le travail et l'interdit qu'il pose, protège de la violence de l'acte sexuel et de la mort. D'un côté, l'un renvoie à la violence introduite par le cadavre dans un monde qu'elle peut ruiner et qui représente un danger pour ceux qui restent, de l'autre, le second s'y oppose en tant que pulsion immédiate qui peut déranger le travail d'une collectivité laborieuse. Bataille résume cette opposition en ces termes : «L'homme est un animal qui demeure "interdit" devant la mort, et devant l'union sexuelle. $»^{44}$

Ce qui nous permet d'affirmer que si les surréalistes choisissent de fuir le monde ordonné par le travail (pour des raisons révolutionnaires ou pas) en choisissant l'exacerbation sans entrave du fait sexuel, alors ils s'ouvrent tout entier à la violence de la mort et d'une certaine façon se trouvent pris en étau entre différentes pulsions qu'ils ne peuvent contrôler et qu'ils redoutent. Cependant, ils tentent de les apprivoiser, non pas en domestiquant l'inconscient mais en le mettant de leur côté en tentant « de vivre en bonne intelligence avec lui $»^{45}$.

\section{Conclusion}

Au terme de cette analyse il convient de résumer quelque peu notre propos. Il s'agissait avant tout de démontrer que bien loin de n'être qu'un roman futile et sans intérêt, Valérie est en réalité une œuvre révélatrice qui permet de toucher, peut-être plus qu'aucune aucune, aux fondements de la pensée surréaliste de Nezval. Bien que tardive, elle synthétise à elle seule les principes ayant provoqué le basculement du poétisme dans le surréalisme. On y retrouve la bestialité attachée au désir sexuel, une plongée infernale dans le psychisme humain menant à un état de doute permanent, le processus automatique symbolisé par la nuit et son érotisme violent qui fascine autant qu'il terrifie. Mais on ne peut comprendre cet expression de l'érotisme qu'en envisageant le rôle primordial que la femme y joue : autre par excellence, objet que l'on modèle et que l'on transforme pour mieux le posséder. La perversité de cette démarche est la clé de lecture du mouvement surréaliste car il serait réducteur et même faux de comprendre ce terme comme traduisant un comportement déviant mais comme une véritable atteinte portée au corps de la société par la destruction du corps humain. Une destruction qui passe par celle du langage, des mots et qui permet d'accéder à la jouissance, à cet état de complétude originel à jamais perdu. 
51 On pourrait alors rappeler que selon Bataille, l'érotisme étant un aspect de la vie intérieure, l'individu n'en prend connaissance que lorsque son expérience personnelle le place de façon égale entre l'interdit et la transgression. L'érotisme naît de ces deux principes inconciliables : le respect de la loi et sa violation. C'est précisément là que se situe le point d'orgue du surréalisme: la recherche d'une traduction la plus fidèle possible de l'expérience intérieure, de ce magma présent en tout homme et qui ne surgit que lorsqu'il est mis sous tension.

La «tension »serait alors le meilleur moyen de comprendre l'acte de création surréaliste. Tension entre tradition et volonté de nouveauté, tension entre réalité sensible et "surréalité » construite par l'esprit, tension paradoxale entre l'eros platonicien et le désir inéducable, entre l'impossible et l'interdit. Nezval œuvre donc dans cet espace où s'affirme le psychique, où il se figure sans jamais trouver d'équilibre stable. Valérie, est incapable de dire si elle rêve ou si elle est consciente, de même qu'elle est incapable de distinguer ses amis de ses ennemis, le vrai, du faux. De la même façon, les poèmes de Nezval se caractérisent par une étrangeté syntaxique et sémantique qui ne permet jamais d'attribuer avec certitude un sens à ses vers. Et c'est sûrement cette absence de stabilité qui fait la force de son écriture. Elle ouvre un gouffre intérieur à la profondeur insondable qui menace l'être de désintégration. L'auteur, touché par le vertige du pouvoir de la création infinie a alors la possibilité de transformer le monde et de le façonner selon sa propre intériorité et d'interagir avec lui, jusqu'à se dissoudre dans cet univers qu'il a lui-même créé: "Ta bouche deux charbons ardents sur lesquels je brûle mes souvenirs ${ }^{46}$. L'écriture surréaliste nezvalienne est en ce sens un perpétuel va et vient entre dedans et dehors, une projection de soi, un don, jusqu'à ce que les frontières de l'esprit deviennent perméables et c'est peut-être là que réside la transgression suprême et originelle du surréalisme.

\section{NOTES}

1. Legrand G., "À propos de la femme-enfant », in: La femme surréaliste, sous la direction de Borderi R., Éd. Borderie, Paris, 1977, p. 9.

2. Breton A., Catalogue de l'exposition internationale du surréalisme de 1959, Galerie Daniel Cordier, Paris, 1959, p. 7 (Les textes du catalogue sont principalement d'André Breton, Hans Bellmer, JeanPierre Duprey, Joyce Mansour, Leonora Carrington, Robert Lebel, Alain Joubert, Radovan Ivšić, etc. Il est illustré par des œuvres de Toyen, Man Ray et Gilles Ehrmann).

3. Bataille G., L'Érotisme, Les Éditions de minuit, Paris, 1957, 312 p.

4. Brouk B., Psychoanalytická Sexuologie (Sexologie psychanalytique), Alois Srdce, Prague, 1933, 169

p.

5. Nezval V, Sexuální nocturno: př́běh demaskované iluse (Nocturne Sexuel : histoire d'une illusion démasquée), Torst, Prague, 2001, 60 p.

6. «Edice 69 » (Édition 69) est un supplément à la Erotická revue (Revue érotique), publiée par Jindřich Štyrský à raison d'un numéro par an entre 1930 et 1933.

7. Bataille G., op. cit., p. 17. 
8. Idem.

9. Nezval V., «Upír Nosferatu »(Nosferatu le vampire), in : Dílo XXV, Československy Spisovatel, Prague, 1963, p. 465.

10. Nezval V., Valérie et la semaine des merveilles (Avant-propos), Pavillon poche, Robert Laffont, Paris, 2007, p. 7. Les citations sont tirées de la version française. Les prochaines occurences seront notées par [Valérie], suivi du numéro de page.

11. Avant-propos.

12. Nezval V., « Upír Nosferatu »,op. cit., p. 466.

13. Nezval V., « Předmluva $\mathrm{k}$ dosavadnímu dílu » (Prologue à mon œuvre), in : Most (Le Pont),

Fr. Borový, Prague, 1957, 148 p.

14. Ernst M., Rêve d'une petite fille qui voulut entrer au Carmel, J. J. Pauvert, Paris, 1983, pp. 17-19.

15. Ibid., p. 51.

16. Nezval V., « Meteor» (Météore), in : Žena v množném čísle (La Femme au pluriel), Kentaur/ Polygrafia, Prague, 1993, p. 27.

17. Idem.

18. Bataille G., op. cit., p. 17.

19. Idem.

20. Marquis de Sade, cité dans : Ibid., p. 18.

21. Ibid., p. 19.

22. Pontalis Jean-Bertrand, «Introduction », in : Surréalisme et sexualité, Gallimard, Paris, 1971, p. 16.

23. Bataille G., op. cit., p. 100.

24. Ibid., p. 101.

25. Marcuse H., Éros et civilisation, Les Éditions de minuit, Paris, 1963, p. 47.

26. Ibid., p. 90.

27. Bataille G., op. cit., p. 103.

28. Gautier X., Surréalisme et Sexualité, Gallimard, Paris, 1971, p. 67.

29. Artaud A., "Lettres à André Breton ",in :L'Éphémère, $\mathrm{n}^{\circ} 8$, éditions de la fondation Maeght, Paris,

hiver 1968, p. 50

30. Artaud A., "Pour en finir avec le jugement de Dieu »,in : Euvres complètes XIII, Gallimard, Paris, 1974, p. 104.

31. Deleuze G. et Guattari F., Mille plateaux, Les Éditions de minuit, Paris, 2002, p. 197.

32. Artaud A., « Pour en finir avec le jugement de Dieu » (Dossier), op. cit.

33. Breton A., « Second manifeste du surréalisme »,in : Manifestes du surréalisme, Gallimard, Paris, 1971, p. 78.

34. Cité par Xavière Gautier : op. cit., p. 60.

35. Artaud A., «Le théâtre de la cruauté » in : CEuvres complètes XIII, op. cit., p. 116.

36. Pontalis J-B., Vocabulaire de la psychanalyse, PUF, Paris, 2004, p. 307.

37. Ibid., p. 130.

38. Nezval V., « Píseň písní » (Le Cantique des cantiques) in : Žena v množném čísle (La Femme au pluriel), Kentaur/Polygrafia, Prague, 1993, p. 15.

39. Svanberg M. W., Catalogue de l'Exposition internationale du surréalisme, Galerie Daniel Cordier, Paris, 1959, p. 45.

40. Nezval V., « Píseň písní », op. cit., p. 18.

41. Pontalis J.-B., Surréalisme et sexualité (Introduction), Gallimard, Paris, 1971, p. 17.

42. Gautier X., op. cit., p. 266.

43. Bataille G., op. cit., p. 48.

44. Ibid., p. 57.

45. Pontalis J-B, op. cit., p. 17. 
46. Nezval V., « Píseň písní », op. cit., p. 16

\section{INDEX}

Index chronologique : communisme, Deuxième Guerre mondiale, entre-deux-guerres Mots-clés : littérature tchécoslovaque, littérature tchèque, poétisme tchèque oeuvretraite Nezval V. : Valerie a týden divů

Index géographique : République tchèque, Tchécoslovaquie

\section{AUTEURS}

\section{SOPHIE TEULIĖRE}

Université Paris III - Sorbonne Nouvelle 